\title{
DYNAMICALLY LOADED RIGID-PLASTIC ANALYSIS UNDER LARGE DEFORMATION
}

\author{
$\mathrm{BY}$ \\ YANG GAO \\ Harvard University, Cambridge, Massachusetts \\ Hefei University of Technology. Hefei, P. R. China
}

\begin{abstract}
Extended bounding theorems on maximum deformation and minimum response time are developed for dynamically loaded rigid-plastic structures in the range of large deformations. It is proved that the existence of bounds is directly related to a so-called complementary gap function and its directional-derivative.
\end{abstract}

1. Introduction. Impulsive loading theorems for rigid-plastic structures have been studied for more than 25 years (see Martin et al. [1-7]). But there still exist some theoretical difficulties in attempting to extend the bounding methods to large deformation problems. It is known that bounding theorems in geometrical linear problems are usually derived from the extremal energy principles. However, in finite deformation theory, the extremum properties of both the total potential energy and complementary energy functionals cannot be guaranteed due to geometrical nonlinearity (Gao-Strang, 1988, [9]). So the existence of bounds on maximum displacement and minimum response time for impulsively loaded, rigid-plastic structures is related to the questions of stability (Ploch-Wierzbicki, 1981, [8]). Moreover, by using Lagrangian approach, the plastic yield function depends not only on the Kirchhoff stress tensor, but also on the displacements, which also brings some difficulties in constructing bounding theorems.

Recently, systematic research has been devoted to the geometrical nonlinear mechanics by Gao-Strang (1989). It is proved in [9] that in the finite deformation theory, the extremum property of primal-dual variational problems is determined by a socalled complementary gap function, which plays a key role in nonlinear mechanics [10-13]. The application to impulsive loading problems under moderate large deformation, yields an upper bound theorem for displacement when the gap function has the right sign (see Gao, 1990, [13]).

In the present paper, the bounding theorems on maximum displacement and minimum response time are extended to the cases of arbitrary large deformations. We will see that the existence of the bounds is directly related to the property of the gap function and its directional-derivative.

Received October 20, 1989.

Supported in part by National Science Foundation of P. R. China for Young Scientists.

Author's current address: Department of Mathematics, Harvard University. Cambridge, MA 02138.

(C)1990 Brown University 
2. Governing equations of dynamic problems. In Euclidean three-dimensional space $R^{3}$, let the reference configuration $\mathscr{B}^{0}$ and current configuration $\mathscr{B}$ of a body be referred to two independent rectangular Cartesian coordinate systems: Lagrange system $\left\{X_{\alpha}\right\}$ and Euler system $\left\{x_{i}\right\}$, respectively. The corresponding basis vectors are $\mathbf{G}_{\alpha}$ and $\mathbf{g}_{i}$. The deformation of the body from $\mathscr{B}^{0}$ to $\mathscr{B}$ may be described as

$$
\mathbf{x}=\mathbf{x}(\mathbf{X})=\mathbf{X}+\mathbf{u}(\mathbf{X}) \text {. }
$$

We adopt the gradient notation $\nabla=\left(\partial / \partial X_{\alpha}\right) \mathbf{G}_{\alpha}$ in reference configuration $\mathscr{B}^{0}$, and $\nabla_{r}=\left(\partial / \partial x_{i}\right) \mathbf{g}_{i}$ in current configuration $\mathscr{B}$, then the deformation gradient tensor may be written as

$$
\mathbf{D}=\mathbf{x} \nabla=\frac{\partial x_{i}}{\partial X_{\alpha}} \mathbf{g}_{i} \otimes \mathbf{G}_{\alpha}=\mathbf{I}+\mathbf{u} \nabla=D_{i, \alpha} \mathbf{g}_{i} \otimes \mathbf{G}_{\alpha} .
$$

For an admissible deformation, $\mathbf{D}$ is nonsingular and finite, then we have the inverse of $\mathbf{D}$ :

$$
\mathbf{D}^{-1}=\mathbf{X} \nabla_{r}=\frac{\partial X_{r r}}{\partial x_{i}} \mathbf{G}_{a r} \otimes \mathbf{g}_{i}=\mathbf{I}-\mathbf{u} \nabla_{r} .
$$

Let $\Omega$ be an open, bounded, connected subset of $R^{3}$ with Lipschitz boundary $\Gamma=\Gamma_{t} \cup \Gamma_{v} . \mathscr{U}$ is the admissible displacement space. For any given $\mathbf{u} \in \mathscr{U}$, the Green strain tensor is denoted by the following geometrical relations:

$$
\begin{aligned}
\mathbf{E} & :=\frac{1}{2}[\nabla \mathbf{u}+\mathbf{u} \nabla+(\nabla \mathbf{u})(\mathbf{u} \nabla)]=\frac{1}{2}\left[\mathbf{D}^{T} \mathbf{D}-\mathbf{I}\right] \\
& =\frac{1}{2}\left[u_{\alpha, \beta}+u_{\beta, \alpha}+u_{\alpha, \gamma} u_{\gamma, \beta}\right] \mathbf{G}_{\alpha} \otimes \mathbf{G}_{\beta} .
\end{aligned}
$$

The abstract description of Eq. (4) can be given as [9]:

$$
\mathbf{E}=A(\mathbf{u}) \mathbf{u}
$$

where $A: \mathscr{U} \rightarrow \mathscr{E}$ is a geometrical nonlinear operator, $\mathscr{E}$ is the admissible strain space. The directional-derivative of $\mathbf{E}$ at $\mathbf{u}$ in the direction $\mathbf{v} \in \mathscr{U}$ is defined as:

$$
\delta \mathbf{E}(\mathbf{u} ; \mathbf{v}):=\lim _{\theta \rightarrow 0^{+}} \frac{\mathbf{E}(\mathbf{u}+\theta \mathbf{v})-\mathbf{E}(\mathbf{u})}{\theta}=A_{t}(\mathbf{u}) \mathbf{v},
$$

where $A_{t}: \mathscr{U} \rightarrow \mathscr{E}$ is called the Gâteaux derivative (cf., e.g., $\left.[15,16]\right)$ of $\mathbf{E}(\mathbf{u})$ at $\mathbf{u}$, which is also a nonlinear geometric mapping:

$$
A_{t}(\mathbf{u}) \mathbf{v}=\frac{1}{2}[\nabla \mathbf{v}+\mathbf{v} \nabla+(\nabla \mathbf{v})(\mathbf{u} \nabla)+(\nabla \mathbf{u})(\mathbf{v} \nabla)] .
$$

Its complementary operator is defined as

$$
A_{n}(\mathbf{u}) \mathbf{v}:=-\frac{1}{4}[(\nabla \mathbf{v})(\mathbf{u} \nabla)+(\nabla \mathbf{u})(\mathbf{v} \nabla)]
$$

So the material derivative of $\mathbf{E}$ may be written as

$$
\begin{aligned}
\dot{\mathbf{E}}(\mathbf{u}) & =A_{t}(\mathbf{u}) \dot{\mathbf{u}}=\mathbf{D}^{T}(\mathbf{u}) \mathbf{d}(\dot{\mathbf{u}}) \mathbf{D}(\mathbf{u}) \\
& =\frac{1}{2}[\nabla \dot{\mathbf{u}}+\dot{\mathbf{u}} \nabla+(\nabla \mathbf{u})(\dot{\mathbf{u}} \nabla)+(\nabla \dot{\mathbf{u}})(\mathbf{u} \nabla)]
\end{aligned}
$$


where

$$
\mathbf{d}(\dot{\mathbf{u}}):=\frac{1}{2}\left(\nabla_{r} \dot{\mathbf{u}}+\dot{\mathbf{u}} \nabla_{r}\right)=\frac{1}{2}\left(\dot{u}_{i, j}+\dot{u}_{j, i}\right) \mathbf{g}_{i} \otimes \mathbf{g}_{i}
$$

is the rate of deformation tensor.

The stress tensors commonly used in finite deformation theory are Cauchy stress $\sigma \quad\left(=\sigma_{i j} \mathbf{g}_{i} \otimes \mathbf{g}_{j}\right)$; Piola stress; $\tau\left(=\tau_{i \alpha} \mathbf{g}_{i} \otimes \mathbf{G}_{\alpha}\right)$ and Kirchhoff stress: $\mathbf{S} \quad(=$ $\left.S_{\alpha \beta} \mathbf{G}_{\alpha} \otimes \mathbf{G}_{\beta}\right)$. They are interrelated with each other by

$$
\begin{aligned}
& \sigma=j \mathbf{D S} \mathbf{D}^{T}=j \tau \mathbf{D}^{T}, \\
& \tau=\mathbf{D S}=J\left(\mathbf{D}^{-1} \sigma\right), \\
& \mathbf{S}=J\left(\mathbf{D}^{-1} \sigma \mathbf{D}^{-1 T}\right)=\mathbf{D}^{-1} \tau,
\end{aligned}
$$

where $j$ is the volume ratio of volume element before and after deformation, and $J=1 / j$. It is easy to prove that

$$
\sigma: \mathbf{d}=j \mathbf{S}: \dot{\mathbf{E}} \text {. }
$$

The double dot product of two tensors $\mathbf{A}$ and $\mathbf{B}$ means $\mathbf{A}: \mathbf{B}=\operatorname{trace}(\mathbf{A B})$.

Let $\dot{\mathscr{U}}$ be the admissible velocity space, $\mathscr{L}$ the admissible force space. The bilinear form $(*, *): \dot{\mathscr{U}} \times \mathscr{L} \rightarrow \mathscr{R}$ puts $\dot{\mathscr{U}}$ and $\mathscr{L}$ in duality. Let $\dot{\mathscr{E}}$ and $\mathscr{S}$ be the admissible strain rate space, admissible stress space, respectively. The bilinear form $\langle *, *\rangle: \dot{\mathscr{U}} \times S \rightarrow R$ puts $\dot{\mathscr{U}}$ and $\mathscr{S}$ in duality. So the dual pair between $\dot{\mathbf{u}} \in \dot{\mathscr{U}}$ and $\mathbf{t} \in \mathscr{L}$ can be represented as $(\dot{\mathbf{u}}, \mathbf{t})=\dot{\mathbf{u}} \mathbf{t}=\dot{u}_{\alpha} t_{\beta} \mathbf{G}_{\alpha} \mathbf{G}_{\beta}=\dot{u}_{\alpha} t_{\alpha}$. The dual pair of $\dot{\mathbf{E}} \in \dot{\mathscr{U}}$ and $\mathbf{S} \in \mathscr{S}$ is given: $\langle\dot{\mathbf{E}}, \mathbf{S}\rangle=\dot{\mathbf{E}}: \mathbf{S}=\operatorname{tr}(\dot{\mathbf{E}} \mathbf{S})=\dot{E}_{\alpha \beta} S_{\alpha \beta}$. Throughout this paper, the nolations

$$
\begin{gathered}
\langle *, *\rangle_{\Omega}:=\int_{\Omega}\langle *, *\rangle d \Omega \\
(*, *)_{\bar{\Omega}}:=\int_{\Omega}(*, *) d \Omega+\int_{\Gamma}(*, *) d \Gamma
\end{gathered}
$$

are used for convenience. Then the Gauss-Green law for energy integration

$$
\begin{aligned}
\int_{\Omega}\{ & \left.\frac{1}{2}[\nabla \dot{\mathbf{u}}+\dot{\mathbf{u}} \nabla+(\nabla \mathbf{u})(\dot{\mathbf{u}} \nabla)+(\nabla \dot{\mathbf{u}})(\mathbf{u} \nabla)]: \mathbf{S}\right\} d \Omega \\
& =-\int_{\Omega}\left\{\dot{\mathbf{u}}[(\mathbf{I}+\mathbf{u} \nabla) \mathbf{S}] \cdot \nabla d \Omega+\int_{\Gamma} \dot{\mathbf{u}}[(\mathbf{I}+\mathbf{u} \nabla) \mathbf{S}] \mathbf{n} d \Gamma\right.
\end{aligned}
$$

can be written as the following simple form:

$$
\left\langle A_{t}(\mathbf{u}) \dot{\mathbf{u}}, \mathbf{S}\right\rangle_{\Omega}=\left(\dot{\mathbf{u}}, A_{t}^{*}(\mathbf{u}) \mathbf{S}\right)_{\bar{\Omega}}
$$

in which $A_{t}^{*}: \mathscr{P} \rightarrow \mathscr{L}$ is the conjugate operator of $A_{t}$, denoted as:

$$
A_{t}^{*}(\mathbf{u}) \mathbf{S}= \begin{cases}-[(\mathbf{I}+\nabla \mathbf{u}) \mathbf{S}] \cdot \nabla & \text { in } \Omega, \\ (\mathbf{I}+\nabla \mathbf{u}) \mathbf{S n} & \text { on } \Gamma,\end{cases}
$$

where $\mathbf{n}$ is unit normal vector on $\Gamma$, i.e., $A_{t}^{*}$ is just the equilibrium operator. So the equilibrium equations for dynamics problems

$$
\left.\begin{array}{cc}
{[(\mathbf{I}+\mathbf{u} \nabla) \mathbf{S}] \cdot \nabla-\overline{\mathbf{b}}=\rho_{0} \ddot{\mathbf{u}}} & \text { in } \Omega, \\
(\mathbf{I}+\mathbf{u} \nabla) \mathbf{S n}-\overline{\mathbf{t}}=0 & \text { on } \Gamma_{t},
\end{array}\right\}
$$


can be written in an abstract form:

$$
A_{t}^{*}(\mathbf{u}) \mathbf{S}-\overline{\mathbf{f}}=\rho_{0} \ddot{\mathbf{u}} \quad \text { in } \Omega \cup \Gamma_{t},
$$

where $\overline{\mathbf{b}}$ is the body force, $\overline{\mathbf{t}}$ the surface tractions, and

$$
\overline{\mathbf{f}}:= \begin{cases}\overline{\mathbf{b}} & \text { in } \Omega, \\ \overline{\mathbf{t}} & \text { on } \Gamma_{t}, \\ \mathbf{t}(\mathbf{S}, \mathbf{u}) & \text { on } \Gamma_{v} .\end{cases}
$$

For rigid-perfectly plastic media, the constitutive equations in Euler approach are given

$$
\mathbf{d}=\dot{\lambda} \frac{\partial F_{\sigma}(\sigma)}{\partial \sigma}, \quad \dot{\lambda} \geq 0, \quad F_{\sigma}(\sigma) \leq 0, \quad \dot{\lambda} F_{\sigma}(\sigma)=0 \quad \text { in } \Omega .
$$

$F_{\sigma}(\sigma)$ is the plastic yield function, which is assumed to be convex, lower semicontinuous. For Mises material, it takes

$$
F_{\sigma}(\sigma)=\sqrt{\sigma_{i j} \sigma_{i j}}-k,
$$

$k$ is the material parameter. In Lagrange approach, the yield function should be

$$
F_{S}(\mathbf{S}, \mathbf{u})=\sqrt{H_{\alpha \beta \gamma \kappa}(\mathbf{u}) S_{\alpha \beta} S_{\gamma \kappa}}-K=j F_{\sigma}(\sigma)
$$

where $K=J k$,

$$
H_{\alpha \beta \gamma \kappa}=D_{i \alpha} D_{\beta j}^{T} D_{i \gamma} D_{\kappa j}^{T}=H_{\gamma \kappa \alpha \beta}=H_{\beta \alpha \gamma \kappa} .
$$

For any given admissible deformation $\mathbf{D}(\mathbf{u}), F_{S}: \mathscr{S} \rightarrow R$ is convex. The constitutive relations Eq. (19) in Lagrange approach can be written as:

$$
\dot{\mathbf{E}}=\dot{\lambda} \frac{\partial F_{S}(\mathbf{S}, \mathbf{u})}{\partial \mathbf{S}}, \quad \dot{\lambda} \geq 0, \quad F_{S}(\mathbf{S}, \mathbf{u}) \leq 0, \quad \dot{\lambda} F_{S}(\mathbf{S}, \mathbf{u})=0 .
$$

Let $\mathscr{K}$ be a subset of $\mathscr{P} \times \mathscr{U}$ :

$$
\mathscr{K}:=\left\{(\mathbf{S}, \mathbf{u}) \in \mathscr{P} \times \mathscr{U} \mid F_{S}(\mathbf{S}, \mathbf{u}) \leq 0 \text { in } \Omega\right\},
$$

the so-called complementary plastic superpotential $W^{*}: \mathscr{S} \times \mathscr{U} \rightarrow \bar{R}:=R \cup[+\infty]$ is defined as following (see Gao-Strang and Gao-Wierzbicki, 1989, $[10,11]$ ):

$$
W^{*}(\mathbf{S}, \mathbf{u})= \begin{cases}0 & \text { if }(\mathbf{S}, \mathbf{u}) \in \mathscr{K} \\ +\infty & \text { otherwise. }\end{cases}
$$

In the theory of convex analysis, $W^{*}$ is called the indicator of the subset $\mathscr{K}$. For any given $\mathbf{u} \in \mathscr{U}, W^{*}: \mathscr{P} \rightarrow \bar{R}:=R \cup\{+\infty\}$ is convex, lower semicontinuous. Its subdifferential gives the unified form of the constitutive relations Eq. (23)

$$
\dot{\mathbf{E}} \in \partial_{\mathbf{S}} W^{*}(\mathbf{S}, \mathbf{u}) \text {. }
$$

Here $\partial_{\mathbf{S}} W^{*}(\mathbf{S}, \mathbf{u})$ denotes the partial subdifferential of $W^{*}$ for $\mathbf{S}$ at $(\mathbf{S}, \mathbf{u})$, which is a convex subset of $\dot{\mathscr{E}}$ :

$$
\partial_{\mathbf{S}} W^{*}(\mathbf{S}, \mathbf{u})= \begin{cases}\dot{\lambda} \frac{\partial F_{S}(\mathbf{S}, \mathbf{u})}{\partial \mathbf{S}} & \text { if } F_{S}(\mathbf{S}, \mathbf{u})=0, \dot{\lambda} \geq 0 \\ \{0\} & \text { if } F_{S}(\mathbf{S}, \mathbf{u})<0 \\ \varnothing & \text { if } F_{S}(\mathbf{S}, \mathbf{u})>0\end{cases}
$$


For a given $\mathbf{u} \in \mathscr{U}$, the conjugate function of $W^{*}$, i.e., the plastic superpotential $W: \dot{\mathscr{E}} \rightarrow R$ may be obtained by using the Legendre-Fenchel transformation:

$$
\begin{aligned}
W(\dot{\mathbf{E}}, \mathbf{u}) & =\sup _{\mathbf{S} \in S}\left\{\langle\dot{\mathbf{E}}, \mathbf{S}\rangle-W^{*}(\mathbf{S}, \mathbf{u})\right\} \\
& =\sup _{\mathbf{S} \in \not{\not}}\langle\dot{\mathbf{E}}, \mathbf{S}\rangle,
\end{aligned}
$$

i.e., $W$ is the support function of the confex subset $\mathscr{K}$. It is obvious that for a given $\mathbf{u} \in \mathscr{U}, W: \dot{\mathscr{E}} \rightarrow R$ is convex, lower semicontinuous. For Mises material, Eq. (28) gives

$$
W(\dot{\mathbf{E}}, \mathbf{u})=K \sqrt{H_{\alpha \beta \gamma \kappa}^{-1}(\mathbf{u}) \dot{E}_{\alpha \beta} \dot{E}_{\gamma \kappa}} .
$$

Then the inverse form of the constitutive relation (26) can be given as:

$$
\mathbf{S} \in \partial_{\dot{\mathbf{E}}} W(\dot{\mathbf{E}}, \mathbf{u}) \text {. }
$$

Moreover, according to the theory of convex analysis, Eq. (26), Eq. (30) and

$$
\langle\dot{\mathbf{E}}, \mathbf{S}\rangle=W(\dot{\mathbf{E}}, \mathbf{u})+W^{*}(\mathbf{S}, \mathbf{u})=W(\dot{\mathbf{E}}, \mathbf{u})
$$

are equivalent to each others. By performing integration over $\Omega$, using Gauss-Green law, Eq. (31) gives

$$
\begin{aligned}
\int_{\Omega} W\left(A_{t}(\mathbf{u}) \dot{\mathbf{u}}\right) d \Omega & =\left\langle\dot{\mathbf{u}}, A_{t}^{*}(\mathbf{u}) \mathbf{S}\right\rangle_{\bar{\Omega}} \\
& =\int_{\Omega} \dot{\mathbf{u}} \overline{\mathbf{b}} d \Omega+\int_{\Gamma} \dot{\mathbf{u}} \overline{\mathbf{t}} d \Gamma .
\end{aligned}
$$

This is the virtual work principle.

During dynamic deformations, suppose that body $\Omega$ is subjected to an impulsive loading system: surface tractions $\overline{\mathbf{t}}(\mathbf{X}, t)$ on $\Gamma_{t} \times[0, \tau]$ and the impulsive initial velocity field $\dot{\mathbf{u}}_{0}$ in $\Omega$. Therefore, in the case of large deformation, the impulsive loading problem for rigid-perfectly plastic dynamical is that to find field variables $\mathbf{u}, \dot{\mathbf{u}}, \mathbf{S}$ and response time $t_{f}$ such that

(1) Geometric Equation:

$$
\begin{aligned}
& A_{t}(\mathbf{u}) \dot{\mathbf{u}}-\dot{\mathbf{E}}=0 \quad \text { in } \Omega \times\left[0, t_{f}\right] \\
& \mathbf{u}=0, \quad \dot{\mathbf{u}}=0 \quad \text { on } \Gamma_{v} \times\left[0, t_{f}\right]
\end{aligned}
$$

(2) Equilibrium Equation:

$$
\begin{aligned}
& A_{t}^{*}(\mathbf{u}) \mathbf{S}+\rho_{0} \ddot{\mathbf{u}}=0 \text { in } \Omega \times\left[0, t_{f}\right] \\
& A_{t}^{*}(\mathbf{u}) \mathbf{S}-\overline{\mathbf{t}}=0 \text { on } \Gamma_{t} \times\left[0, t_{f}\right]
\end{aligned}
$$

(3) Constitutive Equation:

$$
\dot{\mathbf{E}} \in \partial_{\mathbf{S}} W^{*}(\mathbf{S}, \mathbf{u}) \quad \text { or } \quad \mathbf{S} \in \partial_{\dot{\mathbf{E}}} W(\dot{\mathbf{E}}, \mathbf{u}) \quad \text { in } \Omega \times\left[0, t_{f}\right]
$$

(4) Initial Conditions:

$$
\begin{array}{ll}
\mathbf{u}-\mathbf{u}_{0}=0 & \text { in } \Omega \times[0] \\
\dot{\mathbf{u}}-\dot{\mathbf{u}}_{0}=0 & \text { in } \Omega \times[0]
\end{array}
$$


where $\rho_{0}$ denotes the initial mass density. It is rather difficult to find exact solution for given loading systems, because of double nonlinearity (geometric nonlinearity and constitutive nonlinearity). So the bounding methods for displacements and response times will be useful in engineering application.

3. Lower bound theorem for response time. Let $\dot{\mathscr{U}}_{a}$ be a time-independent kinematically admissible space:

$$
\dot{\mathscr{U}}_{a}:=\left\{(\dot{\mathbf{v}}, \mathbf{v}) \subset \dot{\mathscr{U}} \times \mathscr{U} \mid \mathbf{v}=\dot{\mathbf{v}}=0 \text { on } \Gamma_{l}\right\} .
$$

Introducing the so-called gap function [9] $G: \mathscr{U} \times \mathscr{S} \rightarrow \bar{R}$ :

$$
G(\mathbf{v}, \mathbf{T}):=\left\langle-A_{n}(\mathbf{v}) \mathbf{v}, \mathbf{T}\right\rangle_{\Omega}=\int_{\Omega} \frac{1}{2}[(\nabla \mathbf{v})(\mathbf{v} \nabla)]: \mathbf{T} d \Omega .
$$

The directional-derivative of $G$ at $(\mathbf{v}, \mathbf{T})$ in the direction $\dot{\mathbf{v}}$ is:

$$
\delta G(\mathbf{v}, \mathbf{T} ; \dot{\mathbf{v}})=\left\langle-2 A_{n}(\mathbf{v}) \dot{\mathbf{v}}, \mathbf{T}\right\rangle_{\Omega} .
$$

One can propose the following lower bound theorem for response time $t_{f}$ :

TheOREM 1. Suppose $\mathbf{u}, \dot{\mathbf{u}}, \mathbf{S}$ solves the boundary-initial value problem (33). For any given time-independent kinematically admissible fields $(\dot{\mathbf{v}}, \mathbf{v}) \in \dot{\mathscr{U}}_{a}$, if during the time of motion, $t_{f}$,

$$
\delta G(\mathbf{v}-\mathbf{u}, \mathbf{S} ; \dot{\mathbf{v}})=\left\langle-2 A_{n}(\mathbf{v}-\mathbf{u}) \dot{\mathbf{v}}, \mathbf{S}\right\rangle_{\Omega} \geq 0,
$$

then

$$
t_{f} \geq \frac{\int_{0}^{\tau} d t \int_{\Gamma_{t}} \overline{\mathbf{t}} \dot{\mathbf{v}} d \Gamma+\int_{\Omega} \rho_{0} \dot{\mathbf{u}}_{0} \dot{\mathrm{v}} d \Omega}{\int_{\Omega} W\left(A_{t}(\mathbf{v}) \dot{\mathbf{v}}\right) d \Omega} .
$$

Proof. Since for any given $\mathbf{v} \in \mathscr{U}, W(\dot{\mathbf{E}}, \mathbf{v}): \dot{\mathscr{E}} \rightarrow R$ is convex, so the constitutive relation $\mathbf{S} \in \partial_{\dot{\mathbf{E}}} W(\dot{\mathbf{E}}, \mathbf{u})$ yields

$$
W\left(A_{t}(\mathbf{v}) \dot{\mathbf{v}}\right)-W\left(A_{t}(\mathbf{u}) \dot{\mathbf{u}}\right) \geq\left\langle A_{t}(\mathbf{v}) \dot{\mathbf{v}}-A_{t}(\mathbf{u}) \dot{\mathbf{u}}, \mathbf{S}\right\rangle \quad \forall(\dot{\mathbf{v}}, \mathbf{v}) \in \dot{\mathscr{U}}_{a} .
$$

Integrating inequality (39) over $\Omega$, by using Gauss-Green law, one has

$$
\begin{aligned}
\int_{\Omega} W & \left(A_{t}(\mathbf{v}) \dot{\mathbf{v}}\right) d \Omega \geq\left\langle A_{t}(\mathbf{v}) \dot{\mathbf{v}}, \mathbf{S}\right\rangle_{\Omega} \\
& =\left\langle A_{t}(\mathbf{u}) \dot{\mathbf{v}}, \mathbf{S}\right\rangle_{\Omega}-\left\langle 2 A_{n}(\mathbf{v}-\mathbf{u}) \dot{\mathbf{v}}, \mathbf{S}\right\rangle_{\Omega} \\
& =\left(\dot{\mathbf{v}}, A_{t}^{*}(\mathbf{u}) \mathbf{S}\right)_{\bar{\Omega}}+\left\langle-2 A_{n}(\mathbf{v}-\mathbf{u}) \dot{\mathbf{v}}, \mathbf{S}\right\rangle_{\Omega} \\
& =\langle\dot{\mathbf{v}}, \dot{\mathbf{t}}\rangle_{\Gamma_{1}}-\left\langle\rho_{0} \dot{\mathbf{u}}, \dot{\mathbf{v}}\right\rangle_{\Omega}+\left\langle-2 A_{n}(\mathbf{v}-\mathbf{u}) \dot{\mathbf{v}}, \mathbf{S}\right\rangle_{\Omega} .
\end{aligned}
$$

By performing a time-integration in the interval $\left[0, t_{f}\right]$, noting that the tractions $\mathbf{t}(t)$ only act during $t \leq \tau$, Eq. (40) can be written as

$$
\begin{aligned}
\int_{0}^{t_{f}} d t \int_{\Omega} W\left(A_{t}(\mathbf{v}) \dot{\mathbf{v}}\right) d \Omega \geq & \int_{0}^{\tau}\langle\dot{\mathbf{v}}, \overline{\mathbf{t}}(\mathbf{X}, t)\rangle_{\Gamma_{1}} d t \\
& +\left\langle\rho_{0} \dot{\mathbf{u}}_{0}, \dot{\mathbf{v}}\right\rangle_{\Omega}+\int_{0}^{t_{1}}\left\langle-2 A_{n}(\mathbf{v}-\mathbf{u}) \dot{\mathbf{v}}, \mathbf{S}\right\rangle_{\Omega} d t .
\end{aligned}
$$

For any given $(\mathbf{v}, \dot{\mathbf{v}}) \in \dot{\mathscr{U}}, W\left(A_{t}(\mathbf{v}) \dot{\mathbf{v}}\right)$ is independent of time, and $W\left(A_{t}(\mathbf{v}) \dot{\mathbf{v}}\right) \geq 0$ in $\Omega$. So the left-hand side of Eq. (41) can be written as $t_{f} \int_{\Omega} W\left(A_{t}(\mathbf{v}) \dot{\mathbf{v}}\right) d \Omega$. Dividing both sides of Eq. (41) by $W\left(A_{t}(\mathbf{v}) \dot{\mathbf{v}}\right)$, Theorem 1 is proved if the condition (37) is true. Q.E.D. 
4. Upper bounding theorem on deformation. Let $\mathscr{S}_{a} \subset \mathscr{S} \times \mathscr{U}$ be a time-independent statically admissible space:

$$
\begin{aligned}
\mathscr{S}_{a}:=\left\{(\mathbf{T}, \mathbf{v}) \in \mathscr{S} \times \mathscr{U} \mid A_{t}^{*}(\mathbf{v}) \mathbf{T}-\mathbf{b}=0 \text { in } \Omega\right. \\
\left.A_{t}^{*}(\mathbf{v}) \mathbf{T}-\overline{\mathbf{t}}-\mathbf{t}=0 \text { on } \Gamma_{t}\right\} .
\end{aligned}
$$

Here $\mathbf{b}(\mathbf{X})$ and $\mathbf{t}(\mathbf{X})$ are statically admissible external forces associated with $(\mathbf{T}, \mathbf{v}) \in$ $\mathscr{S}_{a}$.

Denoting $K_{0}$ the initial kinetic energy:

$$
K_{0}:=\int_{\Omega} \frac{1}{2} \rho_{0} \dot{\mathbf{u}}_{0} \dot{\mathbf{u}}_{0} d \Omega
$$

and $u^{D}$ the displacement measure:

$$
u^{D}:=\left\langle\mathbf{b}, \mathbf{u}-\mathbf{u}_{0}\right\rangle_{\Omega}+\left\langle\mathbf{t}, \mathbf{u}-\mathbf{u}_{0}\right\rangle_{\Gamma_{1}}
$$

Then for rigid-perfectly plastic material, the upper bound theorem on maximum displacement may be given below:

THEOREM 2. For any given time-independent statically admissible fields $(\mathbf{T}, \mathbf{v}) \in \mathscr{S}_{a}$, if the gap function satisfies: $G(\mathbf{v}, \mathbf{T}) \geq 0$, then the following inequality holds

$$
u^{D} \leq K_{0}+G\left(\mathbf{v}-\mathbf{u}_{0}, \mathbf{T}\right)+\int_{\Omega} W^{*}(\mathbf{T}, \mathbf{v}) d \Omega .
$$

Proof. Let $(\mathbf{u}, \mathbf{S})$ be the solution of problem (33). According to the convexity of the plastic superpotential $W^{*}(\mathbf{T}, \mathbf{v})$, for a given $\mathbf{v} \in \mathscr{U}$, the subdifferential constitutive relation $\dot{\mathbf{E}}(\mathbf{u}) \in \partial_{\mathbf{S}} W^{*}(\mathbf{S}, \mathbf{u})$ yields the following variational inequality:

$$
W^{*}(\mathbf{T}, \mathbf{v})-W^{*}(\mathbf{S}, \mathbf{u}) \geq\left\langle A_{t}(\mathbf{u}) \dot{\mathbf{u}}, \mathbf{T}-\mathbf{S}\right\rangle \quad \forall \mathbf{T} \in \mathscr{S} .
$$

Let $\mathbf{u}=\mathbf{v}+\delta \mathbf{u}$. Noticing that $\mathbf{v}$ is time-independent, the time derivative gives $\dot{\mathbf{u}}=\delta \dot{\mathbf{u}}$. One has

$$
A_{t}(\mathbf{u}) \dot{\mathbf{u}}=A_{t}(\mathbf{v}) \delta \dot{\mathbf{u}}-2 A_{n}(\delta \mathbf{u}) \delta \dot{\mathbf{u}} .
$$

Substituting into (46), using Gauss-Green transformation, one has

$$
\begin{aligned}
& \int_{\Omega} W^{*}(\mathbf{T}, \mathbf{v}) d \Omega-\int_{\Omega} W^{*}(\mathbf{S}, \mathbf{u}) d \Omega \\
& \geq\left\langle A_{t}(\mathbf{v}) \delta \dot{\mathbf{u}}, \mathbf{T}\right\rangle_{\Omega}-\left\langle 2 A_{n}(\delta \mathbf{u}) \delta \dot{\mathbf{u}}, \mathbf{T}\right\rangle_{\Omega}-\left\langle A_{t}(\mathbf{u}) \delta \dot{\mathbf{u}}, \mathbf{S}\right\rangle_{\Omega} \\
&=\left\langle\delta \dot{\mathbf{u}}, A_{t}^{*}(\mathbf{v}) \mathbf{T}-A_{t}^{*}(\mathbf{u}) \mathbf{S}\right\rangle_{\bar{\Omega}}-\left\langle 2 A_{n}(\delta \mathbf{u}) \delta \dot{\mathbf{u}}, \mathbf{T}\right\rangle_{\Omega} \quad \forall(\mathbf{T}, \mathbf{v}) \in \mathscr{S} \times \mathscr{U} \\
&=\langle\mathbf{b}, \delta \dot{\mathbf{u}}\rangle_{\Omega}+\langle\mathbf{t}, \delta \dot{\mathbf{u}}\rangle_{\Gamma_{t}}+\left\langle\rho_{0} \ddot{\mathbf{u}}, \delta \dot{\mathbf{u}}\right\rangle_{\Omega} \\
&-\left\langle 2 A_{n}(\delta \mathbf{u}) \delta \dot{\mathbf{u}}, \mathbf{T}\right\rangle_{\Omega} \quad \forall(\mathbf{T}, \mathbf{v}) \in \mathscr{S}_{a} .
\end{aligned}
$$

By performing the time integration in the interval $\left[0, t_{f}\right]$, noticing that $\mathbf{v}, \mathbf{T}$ are time-independent, it is easy to obtain

$$
\begin{aligned}
\int_{0}^{t_{t}} \int_{\Omega} W^{*}(\mathbf{T}, \mathbf{v}) d \Omega d t-\int_{0}^{t_{t}} \int_{\Omega} W^{*}(\mathbf{S}, \mathbf{u}) d \Omega d t \\
\geq u^{D}+\frac{1}{2} \int_{\Omega} \rho_{0} \dot{\mathbf{u}} \mathbf{u} d \Omega-K_{0} \\
\quad+G(\mathbf{u}-\mathbf{v}, \mathbf{T})-G\left(\mathbf{u}_{0}-\mathbf{v}, \mathbf{T}\right) \quad \forall(\mathbf{T}, \mathbf{v}) \in \mathscr{S}_{a} .
\end{aligned}
$$


For a given $(\mathbf{T}, \mathbf{v}) \in \mathscr{S}_{a}$, if condition $G(\mathbf{v}, \mathbf{T}) \geq 0$ holds, we have $G(\mathbf{u}-\mathbf{v}, \mathbf{T}) \geq 0$. Omitting the nonnegative terms in the right-hand side of Eq. (49) and noticing the property $(25)$ of the superpotential $W^{*}$, the theorem is easily proved. Q.E.D.

5. Complementary optimal bounds. Let $t^{-}: \dot{\mathscr{U}}_{a} \rightarrow \bar{R}$ be a lower bound of response time $t_{f}$ :

$$
t^{-}(\dot{\mathbf{v}}, \mathbf{v}):=\frac{\int_{0}^{\tau} d t \int_{\Gamma_{t}} \overline{\mathbf{t}} \dot{\mathbf{v}} d \Gamma+\int_{\Omega} \rho_{0} \dot{\mathbf{u}}_{0} \dot{\mathbf{v}} d \Omega}{\int_{\Omega} W\left(A_{t}(\mathbf{v}) \dot{\mathbf{v}}\right) d \Omega} .
$$

According to Theorem 1, an optimal lower bound approximation on $t_{f}$ can be given as:

$$
\begin{gathered}
t_{f} \geq \sup _{(\dot{\mathbf{v}}, \mathbf{v}) \in \dot{\mathscr{U}}_{a}} t^{-}(\dot{\mathbf{v}}, \mathbf{v}) \\
\text { s.t. } \delta G(\mathbf{v}-\mathbf{u}, \mathbf{S} ; \dot{\mathbf{v}}) \geq 0 \quad \forall(\dot{\mathbf{v}}, \mathbf{v}) \in \dot{\mathscr{U}}_{a} .
\end{gathered}
$$

On the opposite side, let $u^{+}: \mathscr{S}_{a} \rightarrow \bar{R}$ be an upper measure of the displacement $u^{D}$ :

$$
u^{+}(\mathbf{T}, \mathbf{v}):=K_{0}+G\left(\mathbf{v}-\mathbf{u}_{0}, \mathbf{T}\right)+\int_{\Omega} W^{*}(\mathbf{T}, \mathbf{v}) .
$$

Then according to Theorem 2, an optimal upper approximation of $u^{D}$ can be given as:

$$
\begin{gathered}
u^{D} \leq \inf _{(\mathbf{T}, \mathbf{v}) \in \mathcal{J}_{a}} u^{+}(\mathbf{T}, \mathbf{v}) \\
\text { s.t. } G(\mathbf{v}, \mathbf{T}) \geq 0, \quad \forall(\mathbf{T}, \mathbf{v}) \in \mathscr{F}_{a} .
\end{gathered}
$$

This a nonlinear optimization problem with two variables defined on the feasible set $\mathscr{S}_{a}$. According to various constructions of the plastic superpotential $W^{*}$, (see Gao, 1988, [17, 18]), different variational approximations may be given to solve the problem (53).

Theorems 1, 2 given above show that in impulsively loaded plastic systems under finite dynamic deformation, the existence of bounds on maximum displacement and minimum response time are determined by the property of the gap function:

$$
G(\mathbf{v}, \mathbf{T})=\int_{\Omega} \frac{1}{2} v_{\alpha, \gamma} v_{;, \beta} T_{\alpha \beta} d \Omega,
$$

and its directional-derivative $\delta G(\mathbf{v}, \mathbf{T} ; \dot{\mathbf{v}})$. In the geometrical nonlinear structural analysis, this gap function also provides a global extremum creteria for the dualcomplementary variational problems (see $[19,10])$. In one-dimensional beam bending problem, the gap function will degeneralize to the following form (see [11]):

$$
G(w, N)=\int_{0}^{L} \frac{1}{2}\left(w_{. x}\right)^{2} N(x) d x,
$$

where $w$ is deflection of the beam, and $N$ is axial force. It is obviously that if $N(x) \geq 0 \quad \forall x \in[0, L]$, the gap function $G(w, N) \geq 0 \forall w(x)$. In this case, the structure is stable. Theorem 2 assures that there exists an upper bound on deflective for impulsively loaded plastic beam. Otherwise, the structure may be unstable. 


\section{REFERENCES}

[1] J. B. Martin, A displacement bound technique for elastic continua subjected to a certain class of dynamic loading, J. Mech. Phys. Solids 12, 165 (1964)

[2] J. B. Martin, The determination of upper bounds on displacements resulting from static and dynamic loading by the application of energy methods, IVth National U.S. Congress of Applied Mechanics, 1966, p. 221

[3] A. R. S. Ponter, An upper bound on the displacements of elastic-perfectly plastic structures, J. Appl. Mech. 39, 959 (1972)

[4] W. J. Stronge, Lower bound on deformation for dynamically loaded rigid-plastic structures, Internat. J. Solids and Structures 19, 1049-1063 (1983)

[5] G. Maier and L. Corradi, Upper bounds on dynamic deformations of elastoplastic continua, Meccanica 930 (1974)

[6] M. Capurso, Extended displacements bound theorems for continua subjected to dynamic loading, J. Mech. Phys. Solids 23, 113 (1975)

[7] T. Wierzbicki, Bounds on large dynamic deformations of structures, J. Eng. Mech. Div. Proc. ASCE EM, 267-276 (1970)

[8] J. Ploch and T. Wierzbicki, Bounds for large plastic deformations of dynamically loaded continua and structures, Internat. J. Solids and Structures 17, 183-195 (1981)

[9] Yang Gao and G. Strang, Geometric nonlinearity: Potential energy, complementary energy and the gap function, the 17th International Congress of the Theoretical and Applied Mechanics, Grenoble, France, 1988 (Quart. Appl. Math. 48, 487-504 (1989)

[10] Yang Gao and Gilbert Strang, Dual extremum principles in finite deformation elastoplastic analysis, Acta Appl. Math. 17 257-267 (1989)

[11] Yang Gao and Tomasz Wierzbicki, Bounding theorem in finite plasticity with hardening effect, Quart. Appl. Math. 48 395-403 (1989)

[12] Yang Gao, Opposite principles in nonlinear conservative systems, 4th Int. Conf. on System Research, Informatics and Cybernetics, Baden-Baden, W. Germany, 1988 (Adv. in Appl. Math. (3) 10 370-377 (1989))

[13] Yang Gao, Bounding theorem on finite dynamic deformations of plasticity, Mech. Research Commu. (1) 17, 33-39 (1990)

[14] Yang Gao, Extended dual bounding theorems for nonlinear plastic limit analysis, to appear in Internat. J. Solids and Structures 1990

[15] I. Ekeland and R. Temam, Convex Analysis and Variational Problems, North-Holland, 1976

[16] P. D. Panagiotopoulos, Inequality Problems in Mechanics and Applications, Birkhäuser, 1985

[17] Yang Gao, (1988) On the complementary bounding theorems of limit analysis, Internat. J. Solids and Structures 24 545-556 (1988)

[18] Yang Gao, Panpenalty finite element programming for limit analysis, Comput. and Structures 28, 749-755 (1988)

[19] Yang Gao and Y. K. Cheung, On the extremum complementary energy principles for geometrical nonlinear thin elastic shell, Internat. J. Solids and Structures 26 (5-6), 683-693 (1990)

[20] Yang Gao, On the extreme variational principles for nonlinear elastic plates, Quart. Appl. Math. 48, 361-370 (1990) 